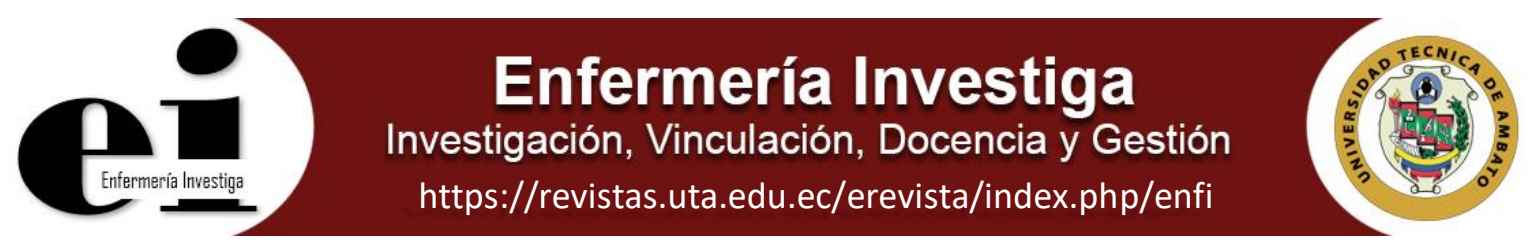

\title{
EDITORIAL
}

\section{RED IBEROAMERICANA DE INVESTIGACION EN CIENCIAS DE LA SALUD}

\section{IBEROAMERICAN RESEARCH NETWORK IN HEALTH SCIENCES}

\begin{abstract}
Jorymar Leal Montiel, $\mathrm{PhD}^{1}$
${ }^{1}$ Docente Titular de la Catedra de Inmunología, Departamento de Ciencias Fisiológicas. Miembro del Instituto de Investigaciones Biológicas. Directora de la División de Investigación de la Facultad de Medicina. Coordinadora de la Maestría de Inmunología Experimental. Docente del Programa de Doctorados en Ciencias de la Salud y Ciencias Médicas de la División de Estudios para Graduados de la Facultad de Medicina de la Universidad del Zulia - Venezuela
\end{abstract}

ORCID: https://orcid.org/0000-0002-1110-9824

Correo electrónico:jorymarleal@gmail.com, jylealluz@yahoo.com

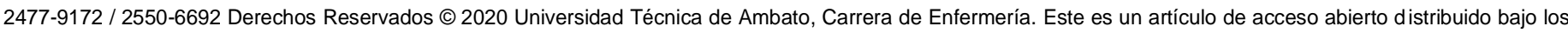

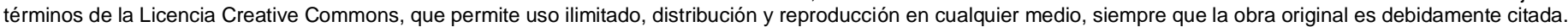

Recibido: 10 de septiembre 2020

Aceptado: 20 de octubre 2020

Las redes de investigación surgen con el avance y desarrollo de la ciencia en el siglo XX, a partir de los cambios generados en la sociedad por los canales de distribución y difusión de las tecnologías de la información y de la comunicación (TIC) en todas las regiones del mundo y especialmente en América Latina. En este contexto, las redes electrónicas de investigación y desarrollo $(\mathrm{I}+\mathrm{D})$ provienen de la evolución de la sociedad del conocimiento en el inicio de las redes en el sistema social o la llamada sociedad en red (1). En este sentido, a mediados de los años noventa en América Latina, se apreciaron los primeros cambios en las políticas de ciencia y tecnología que fortalecieron el desarrollo de las redes de $I+D$ concentrados en la reorganización institucional de la gestión en los sistemas de ciencia y tecnología, así como en las modificaciones introducidas por las legislaciones que daban a los Estados una mayor responsabilidad en el fomento de las actividades de I+D, la integración de las universidades y los centros de investigación en dicha estrategia (2).

En el siglo XXI, en el marco de una sociedad globalizada, las redes de investigación juegan un papel importante como productoras de conocimientos e intercambio y transmisión de los mismos, partiendo de la interacción social, uno de los principales factores determinantes de la globalización de la ciencia, es la disponibilidad de los socios potenciales y la disposición a colaborar en las actividades de investigación conjuntas $(3,4)$. Las redes de investigación se definen como asociaciones de grupos para actividades de investigación y desarrollo tecnológico a partir de programas y proyectos de investigación, orientadas al logro de resultados comunes provenientes de una política de desarrollo científico, social, tecnológico e innovativo en el área del conocimiento humano (5).

Sin embargo, las redes de investigación van más allá de la simple interconexión temática e informativa por parte de grupos de investigadores sobre un tema en particular, constituyen una estrategia para el desarrollo de los sistemas de ciencia y tecnología a escala mundial, como mecanismo de apoyo inter y transdisciplinario en el avance de la ciencia (6). En este ámbito, las redes de investigación se sustentan en las distintas organizaciones de producción de conocimiento como las universidades y centros especializados en investigación tanto en el área pública como privada. De este modo, las universidades de América Latina y el Caribe han logrado tener protagonismo en el desarrollo de las capacidades científicas y tecnológicas de la región $(7,8)$.

En el año 2019, la Universidad Técnica de Ambato (UTA), Facultad de Ciencias de la Salud, Carrera Enfermería realizó el "III Congreso Internacional de Avance en Enfermería e Investigación" del 09 al 13 de diciembre de 2019, evento científico de alta concurrencia de toda la comunidad universitaria. Durante esta actividad científica se enfatizó la importancia de la investigación como fuente generadora de nuevos saberes y propiciadora de soluciones a los problemas de salud en el entorno, cabe destacar, las autoridades académicas de la institución revelaron la necesidad de fortalecer la investigación en cumplimiento con lo establecido en el Estatuto de la UTA, artículo 9 que señala "La Universidad Técnica de Ambato tendrá los siguientes fines: a) Aportar al desarrollo del pensamiento universal, a la producción científica, la innovación y la transferencia tecnológica para lo cual, el Vicerrectorado de investigación, innovación emprendimiento y vinculación con la sociedad basado en sus atribuciones señalada en el artículo 39 literal a) "Planificar, organizar, coordinar y dirigir las actividades de investigación, desarrollo tecnológico, innovación y vinculación con la sociedad" y literal k) "Celebrar contratos y convenios específicos en el ámbito de investigación, desarrollo e innovación y vinculación con la sociedad" (9). 
Por ello, con el fin de establecer alianzas de cooperación la Universidad Técnica de Ambato promovió reuniones estratégicas con investigadores de los países invitados: Venezuela, México y Perú, concluyendo en la necesidad de conformar la Red para la promoción y difusión de la investigación en el área de las Ciencias de la Salud. Surge entonces, el diseño de un documento que plasma la intención y el compromiso con la creación la Red Iberoamericana de Investigación en Ciencias de la Salud (REDIICSA) con el objeto de promover el desarrollo de la investigación científica en el área de Ciencias de la Salud en Iberoamérica y el intercambio conjunto de conocimientos y experiencias, para contribuir en el aporte a soluciones a las problemáticas en salud de la región (10). Estará inicialmente conformada por miembros de universidades de los siguientes países: Ecuador (Universidad Técnica de Ambato y Universidad de las Américas), México (Instituto Superior de Estudios de Enfermería del Estado de Chiapas), Perú (Universidad Nacional de Trujillo), Venezuela (Universidad del Zulia) y España (Universidad de las Islas Baleares).

Entre los aspectos centrales que motivan la creación de la REDIICSA se destacan:

1. Construir un espacio de producción, socialización e intercambio del conocimiento de problemas consensuados en el área de Ciencias de la Salud.

2. Fortalecer la relación docencia-investigaciónvinculación a nivel de pregrado y posgrado.

3. Promover la cooperación internacional de la comunidad académica del área Ciencias de la Salud para la producción científica e intercambio de saberes.
4. Hacer visible la productividad científica desarrollada por la comunidad académica de los miembros de la REDIICSA.

5. Promover y facilitar la movilidad de los miembros de la comunidad académica para el desarrollo de programas y proyectos de investigación, en el marco de convenios y acuerdos interinstitucionales.

6. Llevar a cabo actividades de promoción, organización e intercambio del trabajo científico en red mediante eventos, reuniones y medios de divulgación.

Para materializar la propuesta de creación de la REDIICSA-UTA, durante el acto de clausura del "III Congreso Internacional de Avance en Enfermería e Investigación", las autoridades de la Universidad Técnica de Ambato representada por el Dr. Galo Naranjo López (Rector de la Universidad Técnica de AmbatoEcuador), Dra. Elsa Hernández Chérrez (Vicerrectora de Investigación de la Universidad Técnica de Ambato-Ecuador), Dr. Jesús Chicaiza Tayupanta (Subdecano de la Facultad de Ciencias de la Salud de la Universidad Técnica de AmbatoEcuador), MSc. Andrés Chérrez (Universidad Técnica de Ambato-Ecuador) y los miembros internacionales Dra. Amelia Panunzio Rodríguez (Universidad del Zulia-Venezuela), Dra. Jorymar Leal Montiel (Universidad del Zulia-Venezuela) y MSc. Beatriz López Mayorga (Instituto Superior de Estudios de Enfermería del Estado de Chiapas México), firmaron el acuerdo de intención como marco general para la colaboración entre las instituciones, que permitirá el desarrollo de un convenio de cooperación interinstitucional a fin de contribuir con la investigación, el desarrollo tecnológico, la innovación y la vinculación con la sociedad en el área de las Ciencias de la Salud.

\section{Referencias}

1. Royero JD. Las redes de I+D como estrategia de uso de las TIC en las universidades de América Latina. Revista de Universidad y Sociedad del Conocimiento. 2006; 3(2). Disponible en: http://www.uoc.edu/rusc ISSN 1698-580X

2. Sánchez G. Los sistemas de ciencia y tecnología en tensión: Su integración al patrón de reproducción global. Convergencia $\begin{array}{llllll}\text { [artículo en } & \text { línea]. } & \text { 11(35): } & \text { 193-220. } & \text { Disponible }\end{array}$ http://www.uaemex.mx/webvirtual/wwwconver/htdocs/rev35/35pdf/7 GERMAN SANCHEZ.pdf

3. Vera-Muñoz M. Redes de conocimiento un apoyo para grupos de investigación. Revista de Comunicación de la SEECI. 2014 diciembre. Número extraordinario: 9-17. ISSN: 1576-3420 Disponible en: http://dx.doi.org/10.15198/seeci.2014.35E.9-17

4. Ferro R. Tarazona G. Gaona E. Redes de investigación científica: un esfuerzo en el desarrollo de conocimiento en Conjunto. Revista Visión Electrónica. 2013;7(2):185-196.

5. Sebastián J. Las redes de cooperación como modelo organizativo y funcional para la I+D. Redes. 1999;7(15): 97-111.

6. Prada Madrid E. Las redes de conocimiento y las organizaciones. Revista Bibliotecas y tecnologías de la información. 2005 (Octubre - Diciembre); 2(4):16-25. Disponible en: http://eprints.rclis.org/9127/1/redes de conocimiento.pdf

7. Observatorio Iberoamericano de la Ciencia, la Tecnología y la Sociedad (OCTS), dependiente de la OEI, con sede en la Oficina de Buenos Aires. Las universidades, pilares de la ciencia y la tecnología en América Latina., CRES 2018 III Conferencia Regional de Educación Superior. Buenos Aires, junio de 2018;1-47

8. Torres Velandia SA. García Ponce de León O. Redes de investigación y universidad pública. Revista Digital Universitaria. 2008 febrero; 9(2): 1-12 ISSN: 1067-6079 Disponible en http://www.revista.unam.mx/vol.9/num2/art06/int06.htm

9. Marco Legal Universidad Técnica de Ambato (UTA). Disponible en: https://www.uta.edu.ec/v3.2/uta/universidad.html\#marcolegal

10. Chérrez Ramírez A. Carta de Intención. Red Iberoamericana de Investigación en Ciencias de la Salud (REDIICSA) - UTA "III Congreso Internacional de Avance en enfermería e investigación". Facultad de Medicina- Carrera Enfermería. Universidad Técnica de Ambato-Ecuador. 13 diciembre 2019:1-8. 